\title{
PAPER \\ Cell Edge Capacity Improvement by Using Adaptive Base Station Cooperation in Cellular Networks with Fractional Frequency Reuse
}

\author{
Liang XU $^{\dagger \text { a) }}$, Student Member, Koji YAMAMOTO ${ }^{\dagger}$, Hidekazu MURATA ${ }^{\dagger}$, Members, \\ and Susumu YOSHIDA ${ }^{\dagger}$, Fellow
}

\begin{abstract}
SUMMARY The present paper focuses on the application of the base station cooperation (BSC) technique in fractional frequency reuse (FFR) networks. Fractional frequency reuse is considered to be a promising scheme for avoiding the inter-cell interference problem in OFDMA cellular systems, such as WiMAX, in which the edge mobile stations (MSs) of adjacent cells use different subchannels for separate transmission. However, the problem of FFR is that the cell edge spectral efficiency (SE) is much lower than that of the cell center. The BSC technique, in which adjacent BSs perform cooperative transmission for one cell edge MS with the same channel, may improve the cell edge SE. However, since more BSs transmit signals for one cell edge MS, the use of BSC can also increase the inter-cell interference, which might degrade the network performance. In this paper, with a focus on this tradeoff, we propose an adaptive BSC scheme in which BSC is only performed for the cell edge MSs that can achieve a significant capacity increase with only a slight increase in intercell interference. Moreover, a channel reallocation scheme is proposed in order to further improve the performance of the adaptive BSC scheme. The simulation results reveal that, compared to the conventional FFR scheme, the proposed schemes are effective for improving the performance of FFR networks.
\end{abstract}

key words: base station cooperation, cell edge, fractional frequency reuse, inter-cell interference

\section{Introduction}

Orthogonal frequency-division multiple access (OFDMA) is considered to be a promising technique for downlink transmission in the next generation mobile communication systems, such as 3GPP LTE [1] and WiMAX [2]. One reason for this is that efficient subcarrier allocation enables OFDMA to provide a high data rate, thereby benefiting from multi-user diversity. In addition, the use of OFDMA can also reduce the influence of multi-path channels. However, OFDMA is sensitive to inter-cell interference. In a multicell environment, if the frequency resource is universally reused in each cell of the network, two adjacent cells may use the same subcarrier, which might cause inter-cell interference and severely degrade the cell edge capacity. Meanwhile, traditional frequency reuse schemes, such as the use of a reuse factor of 3 , can reduce the inter-cell interference and improve the cell edge signal-to-interference-plus-noise ratio (SINR). However, the accessible frequency resource is also reduced for each base station (BS), which may decrease the spectral efficiency (SE) of the entire network.

Manuscript received July 21, 2009.

Manuscript revised March 17, 2010.

${ }^{\dagger}$ The authors are with the Graduate School of Informatics, Kyoto University, Kyoto-shi, 606-8501 Japan.

a) E-mail: xuliang@hanase.kuee.kyoto-u.ac.jp DOI: 10.1587/transcom.E93.B.1912
Several schemes of inter-cell interference mitigation are currently being considered in OFDMA networks, such as fractional frequency reuse (FFR) [2] and soft reuse [3], and FFR is considered to be a promising scheme for multicell OFDMA systems. The concept of FFR is based on the partial reuse proposed for time-division multiple access (TDMA) cellular systems [4]. Partial reuse adopts different reuse factors for the cell center and the cell edge. A low reuse factor is adopted for the cell center mobile stations (MSs), whereas a high reuse factor is adopted for the cell edge MSs. This can allow the edge MSs of adjacent cells to use different subchannels for separate transmission without inter-cell interference. Thus, FFR can achieve a much higher network capacity compared to traditional frequency reuse schemes and can simultaneously reduce inter-cell interference compared to a reuse factor of 1 . However, since the cell edges use a higher reuse factor, the cell edge SE may be significantly degraded compared to the cell center. Therefore, it is necessary to increase the cell edge SE, which may be possible using the base station cooperation (BSC) technique.

In the BSC scheme, one BS can transmit signals to MSs of neighbor cells, and adjacent BSs can perform cooperative transmission for one MS using the same channel. It has been shown that the use of BSC can significantly improve the capacity of cell edge MSs [5]-[8]. As yet, no disadvantages of using BSC have been reported. In FFR networks, since different subchannels are allocated to the edge MSs of adjacent cells, the subchannels can be reused by neighbor BSs to realize BSC. However, compared to the conventional FFR scheme, since more BSs transmit signals for one cell edge MS, more inter-cell interference may occur throughout the entire network, which might lead to decreased capacity of other cell edge MSs, and, as a result, the total SE at cell edges may decrease. Therefore, in this paper, focusing on this tradeoff, we first investigate the effect of BSC in FFR networks to show that the use of BSC is not always effective for improving the performance of FFR networks. Based on these findings, we propose an adaptive BSC scheme and a channel reallocation scheme to improve the cell edge SE and the fairness of FFR networks.

The remainder of the present paper is organized as follows. Section 2 describes the FFR scheme and the BSC technique. Section 3 describes a computer simulation to evaluate the performance of BSC in a 19-cell OFDMA network. Section 4 evaluates the proposed adaptive BSC 
scheme and channel reallocation scheme by a computer simulation. Finally, Sect. 5 presents our conclusions and future work.

\section{Fractional Frequency Reuse and Base Station Coop- eration}

In this section, we describe the FFR scheme and the BSC technique.

\subsection{Fractional Frequency Reuse (FFR)}

Fractional frequency reuse [2] is based on the reuse partitioning proposal for TDMA cellular systems [4]. Fractional frequency reuse divides the entire cell into the cell center and the cell edge, and performs time-division transmissions between the cell center and the cell edge in order to achieve high system capacity. For example, as shown in Fig. 1, transmissions of the cell center and cell edge MSs occur during two separate time intervals. In the first time interval, the cell center MSs use the total bandwidth $F_{\text {all }}$ for their transmissions, with a reuse factor of 1 , throughout the entire network, whereas, for the cell edge MSs, the total bandwidth is divided into three subbands: $F_{1}, F_{2}$, and $F_{3}$, and in the second time interval the edge MSs of each cell use only one subband for their transmissions, with a reuse factor of 3 , throughout the entire network. Considering the fairness among cells, it is appropriate to assume that $F_{1}, F_{2}$, and $\mathrm{F}_{3}$ have the same bandwidth.

Using the FFR scheme, cell center MSs that have high SINR can achieve high cell capacity with a short reuse distance. For cell edge users, inter-cell interference can be reduced, and each BS can determine the subchannel allocation freely without any coordination among BSs. However, since the accessible frequency resource of each BS is reduced for cell edge MSs, the cell edge SE may be significantly degraded compared to that of the cell center.

\subsection{Base Station Cooperation}

In conventional cellular systems, one base station transmits signals to the MSs in its own cell without regard to the MSs in adjacent cells. Inter-cell interference can be mitigated by using different frequencies between adjacent cells, but this decreases the spectral efficiency. Therefore, in recent years, the BSC technique, which can increase the cell edge capacity without inter-cell interference, is proposed [7]. As shown in Fig. 2, two adjacent BSs that are connected to a central control unit (CCU) can share the data and transmit signals simultaneously. In this case, $\mathrm{BS}_{1}$ and $\mathrm{BS}_{2}$ can use the subchannel $\mathrm{F}_{1}$ to perform cooperative multiple-input multipleoutput (MIMO) transmission for $\mathrm{MS}_{1}$ that has multiple receiving antennas. This is referred to as the BSC technique. The BSC technique can significantly increase the capacity of cell edge MSs [5]-[8].
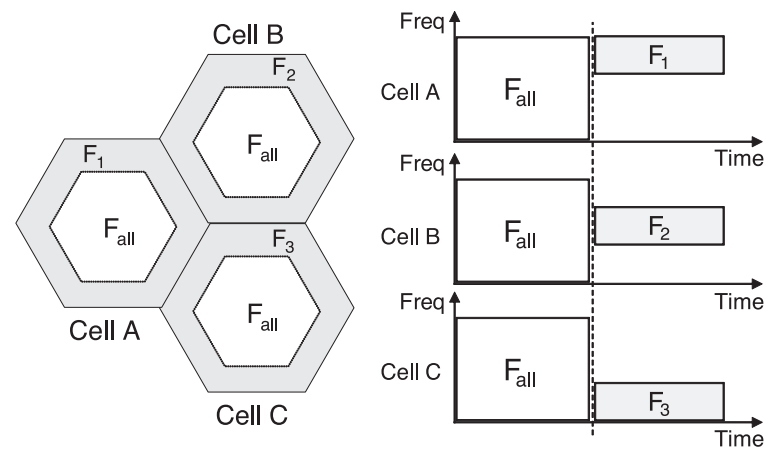

Fig. 1 Fractional frequency reuse scheme.

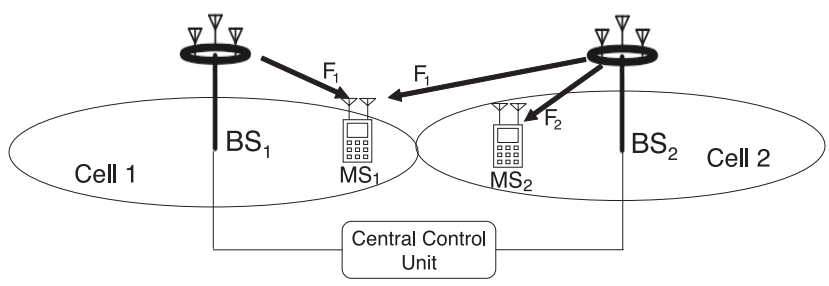

Fig. 2 Base station cooperation.

\section{Performance Evaluation in the Multi-Cell Model}

In this section, we investigate the effectiveness of BSC in multi-cell networks with FFR through a computer simulation. First, the system model and assumptions are described. We then evaluate the cell edge SE, fairness, and average interference power per MS in the system.

\subsection{System Model and Assumptions}

As shown in Fig. 3, we consider a multi-cell network that is composed of 19 cells in which the users are uniformly randomly distributed, and the FFR scheme described in Sect. 2.1 is applied in the network. Each BS uses directional antennas, and each cell is divided into six sectors. The antenna gain of the BS in the direction of the desired sector is $20 \mathrm{~dB}$ higher than that in other directions. For each cell, there is a cell edge area borderline between the BS and the cell edge, which divides the entire cell into two equal-area parts: the cell center and the cell edge, as assumed in [9]. In addition, all of the BSs are connected to a single CCU. There are totally 18 subchannels, and each sector uses three subchannels for the cell center MSs, and one subchannel for the cell edge MSs, as shown in Fig. 3. In the figure, $F_{1,1}$ is one subchannel, and $\mathrm{F}_{\text {all, } 1}$ represents a subband that consists of three subchannels. Each BS has one antenna in each sector, and each MS has two receiving antennas. The transmission power is assumed to be equal for each subcarrier, and one subchannel is defined as a fixed number of subcarriers. In order to achieve the best network performance, the maximum sum rate (MSR) scheme is used for subchannel allocation in the simulation, where the subchannel is allocated 


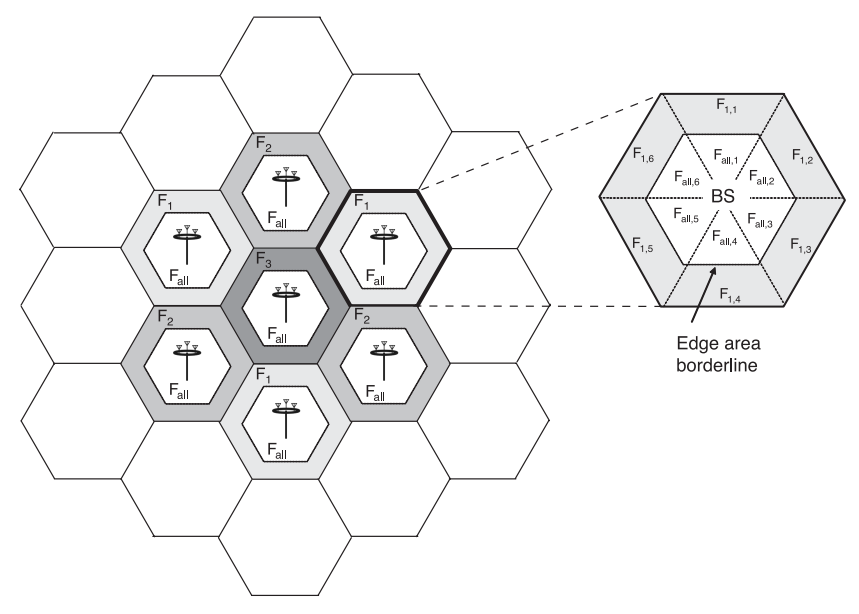

Fig. 3 Cell configuration.

Table 1 Simulation parameters

\begin{tabular}{c|c}
\hline Parameters & Values \\
\hline Number of cells & 19 \\
Number of sectors per cell & 6 \\
Number of MSs per cell & 60 \\
Number of antennas for each MS & 2 \\
Number of antennas for BS in each sector & 1 \\
Number of all subchannels & 18 \\
Path loss exponent & 3.5 \\
Channel model & Rayleigh fading \\
Channel allocation scheme & MSR \\
\hline
\end{tabular}

to the MS with the highest capacity in each sector [10].

When using BSC, for any cell edge MS using a subchannel, the CCU will first find the nearest neighbor BS for the MS according to the signal strength of each BS from the MS. The neighbor BS then checks whether the subchannel to be used is free and informs the CCU as to the status of this subchannel. If the subchannel is free, then the CCU will issue an instruction of cooperative transmission to the two BSs. After that, the neighbor BS will reuse the subchannel of the source BS, and performs cooperative MIMO transmission for the cell edge MS with the source BS, as shown in Fig. 2. Table 1 shows the related simulation parameters. In the simulation, we investigate the capacity performance with different cell edge SNR which is defined as the SNR at the cell edge when the base station transmits a signal without cooperative transmission and any interference. In this case, from the path loss exponent, we can use the cell edge SNR to calculate the ratio of the transmission power to the noise power, which is necessary for calculating the capacity. Therefore, the parameters of the transmission power and the noise power are not necessary here.

\subsection{Modeling of the Downlink Capacity}

Let $h_{1}^{\mathrm{b}}$ and $h_{2}^{\mathrm{b}}$ denote the complex channel gains on either antenna of the MS. When BSC is not used, we assume that maximal ratio combining is performed at the MS. The capacity of the MS can then be expressed as

$$
C_{\text {FFR }}=\log _{2}\left(1+\frac{P\left(\left|h_{1}^{\mathrm{b}}\right|^{2}+\left|h_{2}^{\mathrm{b}}\right|^{2}\right)}{I+N}\right),
$$

where $I$ is the average interference power, $N$ is the noise power, and $P$ is the total transmission power for one subchannel.

When using BSC, the neighbor BS performs cooperative MIMO transmission with the source BS for a cell edge MS using the same subchannel. Here, we assume that the BSs do not have the channel-state information and that both of the BSs use half of the total transmission power. The capacity of the cell edge MS with BSC can be expressed as follows [8]:

$$
C_{\mathrm{BSC}}=\log _{2} \operatorname{det}\left(\boldsymbol{I}_{2}+\frac{P}{2(I+N)} \boldsymbol{A} \boldsymbol{A}^{\mathrm{H}}\right),
$$

where

$$
\boldsymbol{A}=\left[\begin{array}{cc}
h_{1}^{\mathrm{b}} & h_{1}^{\mathrm{c}} \\
h_{2}^{\mathrm{b}} & h_{2}^{\mathrm{c}}
\end{array}\right],
$$

where $\boldsymbol{I}_{2}$ denotes the identity matrix of size $2, h_{1}^{\mathrm{c}}$ and $h_{2}^{\mathrm{c}}$ denote the complex channel gains of cooperative BS.

\subsection{Capacity Fairness}

In this paper, we use the Jain fairness index to measure the fairness among all communicating MSs in the network. The Jain fairness index can be expressed as follows [11]:

$$
F_{\text {Jain }}=\frac{\left(\sum_{i=1}^{n} C_{i}\right)^{2}}{n \sum_{i=1}^{n} C_{i}^{2}},
$$

where $C_{i}$ is the capacity of MS $i$, and $n$ is the total number of the communicating cell center and cell edge MSs. A fairness index of 1 indicates the highest fairness, where all communicating MSs achieve the same capacity. Lower values of the fairness index indicate significant capacity differences among the communicating MSs.

\subsection{Simulation Results}

We investigate the cell edge SE and fairness for different cell edge SNRs. Figures 4, 5, and 6 show the cell edge SE, the average Jain fairness index among all communicating MSs, and the Interference-to-Noise power Ratio (INR) per MS, respectively. In these figures, "FFR" indicates the conventional FFR scheme, whereas "FFR+BSC" indicates the FFR scheme used in conjunction with the BSC technique.

As shown in Fig. 4, when the cell edge SNR is low, BSC cannot improve the cell edge SE compared to the conventional FFR scheme. The reason can be explained as follows:

When the cell edge SNR is low, using BS cooperative MIMO transmission can hardly increase the capacity of the MS. This is because when the average SNR is low, most of the advantage of the MIMO comes only from diversity as mentioned in [12], and the capacity almost depends on the 


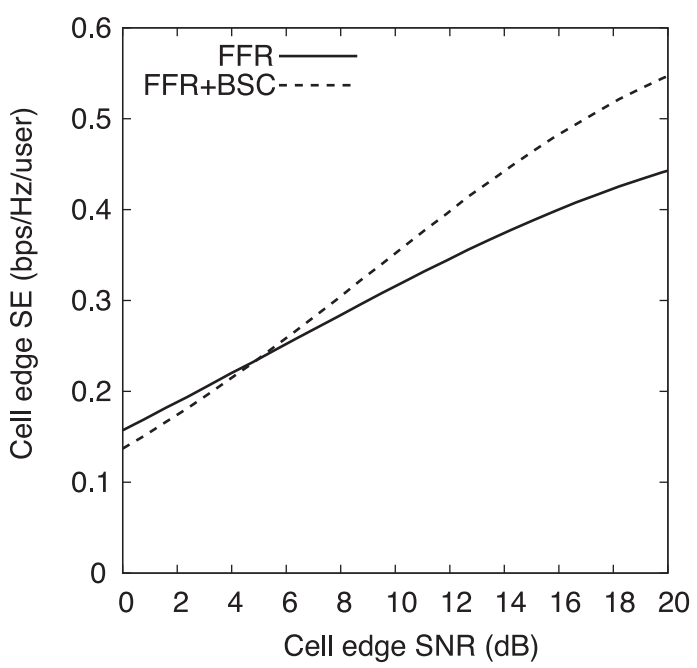

Fig. 4 Cell edge SE.

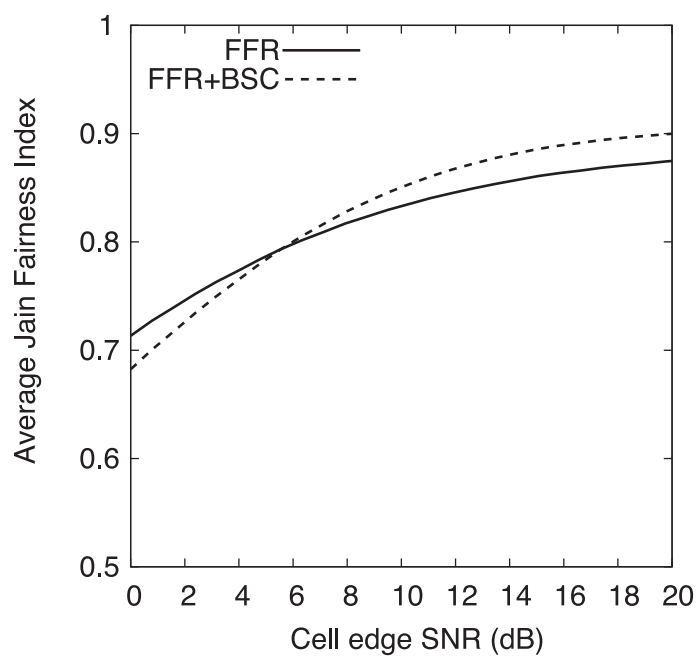

Fig. 5 Fairness.

SNRs from the two BSs. Note that we assume that both of the BSs use half of the total transmission power, thus the total SNR at MS will not increase compared to the case without BSC. Therefore, in this case, using BSC cannot be very effective in increasing the MS's capacity. However, since more BSs transmit signals for one cell edge MS when BSC is used, more inter-cell interference may occur throughout the entire network as shown in Fig. 6, and this may decrease the capacity of other cell edge MSs that use the same subchannel. Therefore, the cell edge SE decreases. However, when the cell edge SNR is high, using MIMO does not only benefit from diversity but also spatial multiplexing as mentioned in [12]. In this case, the increase in capacity achieved by using BS cooperative MIMO transmission is higher than the decrease in capacity caused by the increased inter-cell interference. As such, the cell edge SE increases. Therefore, BSC can improve the cell edge SE, compared to the conventional FFR scheme, only when the cell edge SNR is high.

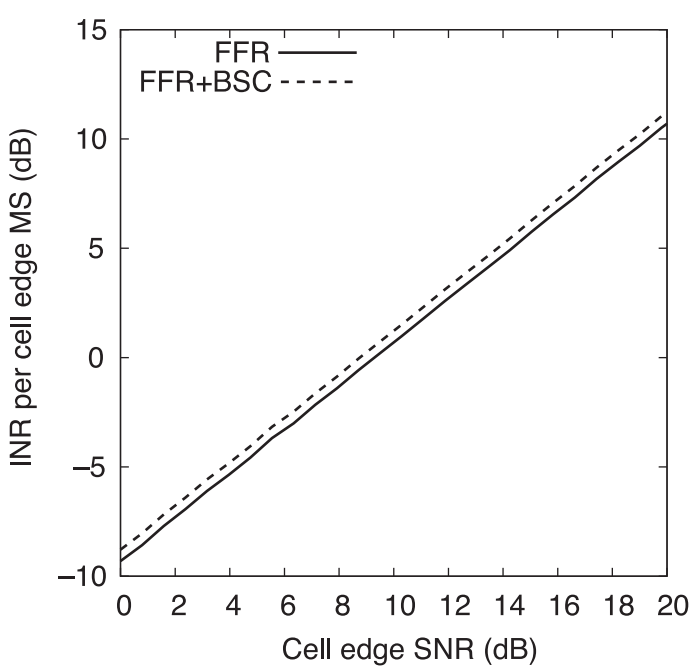

Fig. 6 INR per cell edge MS.

Figure 5 indicates that when the cell edge SNR is high, using BSC can improve the fairness of FFR networks. This is because the fairness index is determined to a large extent by the capacity differential between the cell center and cell edge MSs. When the cell edge SNR is high, BSC can improve the capacity of cell edge MSs, which can reduce the capacity difference among all MSs in the network. However, when the cell edge SNR is low, BSC cannot improve the fairness of FFR networks.

\section{Adaptive BSC in FFR Networks}

In Sect. 3, the simulation results reveal that using BSC does not always improve the cell edge SE, especially when the cell edge SNR is low. Therefore, we next consider an adaptive BSC scheme in which BSC is only performed for some special MSs. In this section, we first describe the proposed adaptive BSC scheme. We then describe subchannel reallocation for the adaptive BSC scheme. Finally, we evaluate the cell edge SE and fairness in order to demonstrate the performance improvement.

\subsection{Adaptive BSC Scheme}

The system model and simulation parameters described in Sect. 3 are also used for the adaptive BSC scheme. Figure 7 illustrates the operational flow chart of the adaptive BSC scheme, and here the subchannels have already been allocated to the MSs in each sector according to the MSR scheme mentioned in Sect. 3.1. For a cell edge MS using a subchannel, the CCU will find the nearest neighbor BS for the MS according to the signal strength of each BS from the MS. The neighbor BS then checks whether the subchannel to be used is free and informs the CCU. In order to investigate the maximum performance of the adaptive BSC scheme, the $\mathrm{CCU}$ is assumed to have perfect knowledge with respect to the capacity of each MS without any overhead. If the subchannel is not free, then the subchannel will 


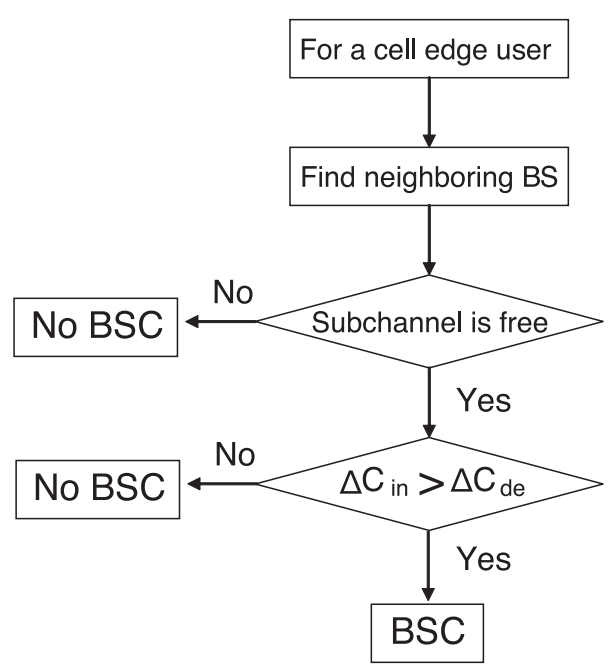

Fig. 7 Flow chart of the adaptive BSC scheme.

not be used for BSC. If the subchannel is free, then the CCU will compare $\Delta C_{\text {in }}$ and $\Delta C_{\mathrm{de}}$, where $\Delta C_{\text {in }}$ denotes the capacity increase of the cell edge MS as a result of using BSC and $\Delta C_{\mathrm{de}}$ denotes the summation of the capacity decrease of all the other cell edge MSs due to the increased inter-cell interference associated with the use of BSC. If $\Delta C_{\mathrm{in}}>\Delta C_{\mathrm{de}}$, then the CCU will control the two BSs to perform cooperative MIMO transmission for the cell edge MS. Otherwise, BSC will not be used.

\subsection{Channel Reallocation for Adaptive BSC}

As mentioned in Sect. 4.1, the subchannels are allocated to the MSs according to the MSR scheme, in order to maximize the cell capacity. However, this conventional channel allocation scheme may not be appropriate for achieving the maximum capacity when using BSC, because the link capacity of the neighbor BS should also be considered for channel allocation. Therefore, in an attempt to further improve the performance of the adaptive BSC scheme, we propose to reallocate the subchannel according to MSR for BSC (referred to hereinafter as MSRB) scheme, in which a subchannel is allocated to the MS that can achieve the maximum capacity with BS cooperative transmission. For example, considering the case that a subchannel has been allocated to $\mathrm{MS}_{\mathrm{a}}$ according to the conventional MSR scheme, and $\mathrm{BSC}$ is selected for $\mathrm{MS}_{\mathrm{a}}$ according to the adaptive BSC scheme described in Sect.4.1. In this case, in the sector that $\mathrm{MS}_{\mathrm{a}}$ is located, there may be another $\mathrm{MS} \mathrm{MS}_{\mathrm{b}}$ that can achieve higher capacity with BS cooperative transmission than $\mathrm{MS}_{\mathrm{a}}$ due to better link quality from the neighbor BS. Therefore, reallocating the subchannel to $\mathrm{MS}_{\mathrm{b}}$ can achieve higher capacity, and this can be realized by using the MSRB scheme. Besides, since the BS's transmission power remains unchanged while reallocating the subchannel, the total inter-cell interference will not increase. Therefore, it is considered that the network performance can be improved by applying channel reallocation for adaptive BSC

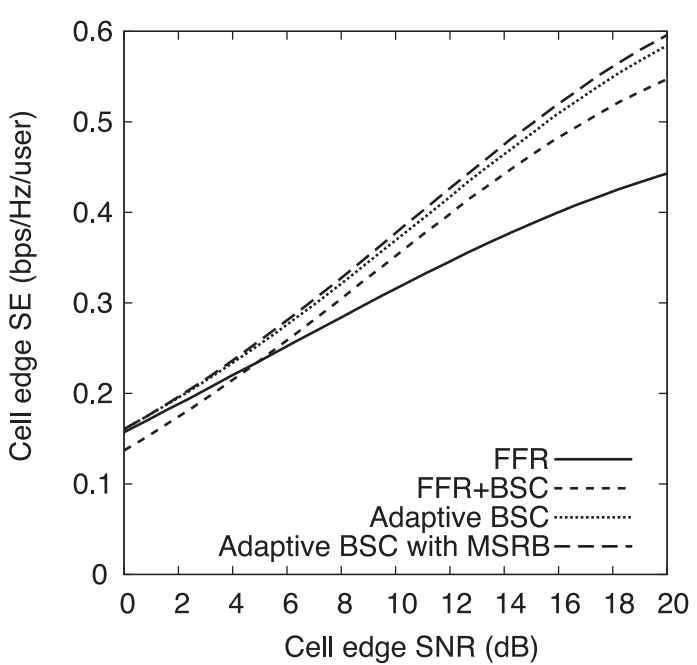

Fig. 8 Cell edge SE (adaptive BSC).

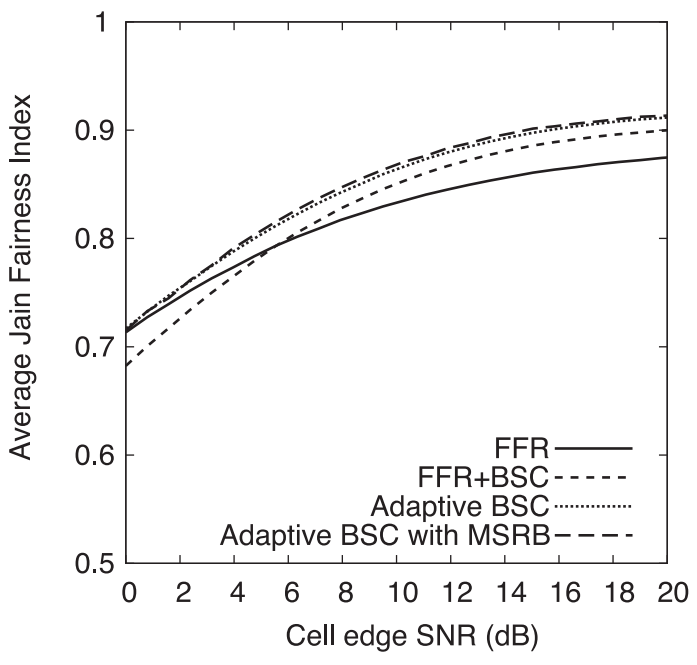

Fig. 9 Fairness (adaptive BSC).

scheme. However, since the communicating $\mathrm{MS}_{\mathrm{a}}$ will lose the subchannel and stop the communication with BS, applying channel reallocation may be difficult in the practical systems.

\subsection{Simulation Results}

Figures 8 and 9 show the cell edge SE and average Jain fairness index among all communicating MSs, respectively. Figure 10 shows the percentage of MSs with BSC in all communicating MSs when using adaptive BSC scheme. In these figures, "FFR," "FFR+BSC," "Adaptive BSC," and "Adaptive BSC with MSRB" indicate the conventional FFR scheme, the BSC scheme, the adaptive BSC scheme, and the adaptive BSC scheme using channel reallocation, respectively.

As shown in Fig. 8, regardless of the cell edge SNR, the adaptive BSC scheme achieves better performance than the conventional FFR scheme and the BSC scheme. This 


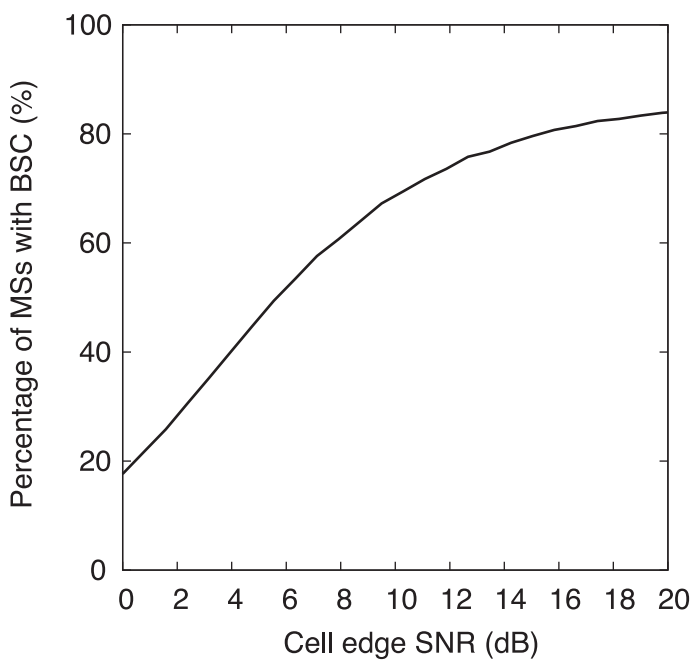

Fig. 10 Percentage of MSs with BSC.

is because the adaptive BSC scheme chooses the better scheme between the conventional FFR scheme and the BSC scheme. When the cell edge SNR is low, since BS cooperative MIMO transmission is not efficient for most MSs, only a few MSs select the BSC scheme, as shown in Fig. 10. However, when the cell edge SNR is high, most MSs select the BSC scheme, because BS cooperative MIMO transmission is very effective in high-SNR environments. Furthermore, although the effect on capacity improvement is not so significant, adaptive BSC with MSRB achieves better performance than adaptive BSC. This is because, unlike conventional MSR, adaptive BSC with MSRB reallocates the subchannel to the MS that can achieve higher capacity when using BSC. However, it may be difficult to apply channel reallocation in practical systems as mentioned in Sect. 4.2. Figure 9 indicates that adaptive BSC and adaptive BSC with MSRB always improve the Jain fairness index, as compared to the conventional FFR scheme. Therefore, the proposed schemes can improve not only the cell edge SE but also the fairness of FFR networks.

However, in practical cellular networks, it is difficult for the CCU to learn $\Delta C_{\text {in }}$ and $\Delta C_{\mathrm{de}}$ immediately. Actually, there should be some overhead, such as feedback from the MSs that use BSC and the MSs that suffer from increased inter-cell interference. Therefore, considering this overhead, the actual performance of the adaptive BSC scheme may be lower than that indicated by the simulation results.

\section{Conclusion and Future Work}

In the present paper, in order to improve the performance of FFR networks, we proposed an adaptive BSC scheme in which BSC is only performed for the cell edge MSs that can achieve a capacity increase with only a slight increase in inter-cell interference. In addition, a channel reallocation scheme, which further improves the performance of the adaptive BSC scheme, was proposed. Simulation results indicate that, compared to the conventional FFR scheme, the proposed schemes are effective for improving the performance of FFR networks.

In this paper, we only consider the case of using constant transmission power for each subchannel. However, using the power control scheme may improve the network performance, which can be recommended as our future work. Besides, the position of the edge area borderline and the way of time division for cell center and cell edge area may also affect the effectiveness of our proposals in FFR networks, which can also be considered as future work.

\section{Acknowledgment}

This work is supported in part by a Grant-in-Aid for Scientific Research (A) (no. 20246067) from the Japan Society for the Promotion of Science (JSPS).

\section{References}

[1] 3GPP TR 25.814, "Physical layer aspects for evolved universal terrestrial radio access (UTRA)," Jan. 2006.

[2] WiMAX Forum, "Mobile WiMAX — Part I: A technical overview and performance evaluation," June 2006.

[3] Huawei, "Soft frequency reuse scheme for UTRAN LTE," 3GPP R1050507, 3GPP TSG RAN WG1 Meeting 41, May 2005.

[4] S.W. Halpern, "Reuse partitioning in cellular systems," Proc. IEEE VTC, pp.322-327, May 1983.

[5] S. Shamai and B.M. Zaidel, "Enhancing the cellular downlink capacity via co-processing at the transmitting end," Proc. IEEE VTC, vol.3, pp.1745-1749, May 2001.

[6] A. Goldsmith, S.A. Jafar, N. Jindal, and S. Vishwanath, "Capacity limits of MIMO channels," IEEE J. Sel. Areas Commun., vol.21, pp.684-702, June 2003.

[7] H. Zhang and H. Dai, "Cochannel interference mitigation and cooperative processing in downlink multicell multiuser MIMO networks," European J. Wireless Commun. and Networking, pp.222235, Dec. 2004.

[8] A. Yoshimoto and T. Hattori, "Prediction of area capacity in a multilink MIMO cellular network with guaranteed QoS,' Proc. IEEE WCNC, pp.1263-1268 March 2007.

[9] S. Uchida, H. Iura, T. Kuze, A. Otsuka, and A. Shibuya, "A study on multi-site cooperative MIMO communication system," Proc. IEICE Gen. Conf. 2009, B-5-43, March 2009.

[10] J.G. Andrews, A. Ghosh, and R. Muhamed, Fundamentals of WiMAX: Understanding Broadband Wireless Networking, Prentice Hall, 2007.

[11] R. Jain, D. Chiu, and W. Hawe, "A quantitative measure of fairness and discrimination for resource allocation in shared computer systems,” DEC Research Report TR-301, Sept. 1984.

[12] J.W. Wallace and M.A. Jensen, "MIMO capacity variation with SNR and multipath richness from full-wave indoor FDTD simulations," Antennas and Propagation Society International Symposium, vol.2, pp.523-526, June 2003. 


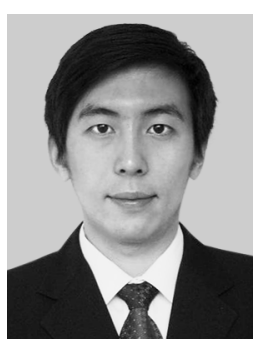

Liang Xu received the B.E. degree in electrical and electronic engineering from Kyoto University in 2005, and the M.E. degree from Graduate School of Informatics at Kyoto University in 2007 . He is currently studying towards his Ph.D. degree as a Japanese Government Scholarship student at Kyoto University. He received the IEICE RCS Active Research Award and IEEE Kansai Student Researcher Encouragement Award in 2007 and 2010, respectively.

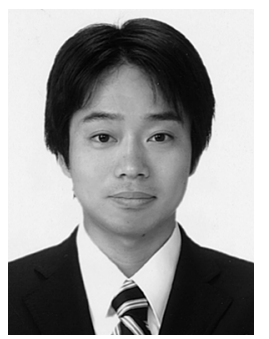

Koji Yamamoto received the B.E. degree in electrical and electronic engineering from Kyoto University in 2002, and the M.E. and Ph.D. degrees in informatics from Kyoto University in 2004 and 2005, respectively. Since 2005, he has been an assistant professor of the Graduate School of Informatics, Kyoto University. From 2008 to 2009 , he was a visiting researcher at Wireless@KTH, Royal Institute of Technology (KTH) in Sweden. His research interests include game theory, spectrum sharing, and cooperative multi-hop networks. He received the PIMRC 2004 Best Student Paper Award in 2004, the Ericsson Young Scientist Award in 2006, and the Young Researcher's Award from the IEICE of Japan in 2008. He is a member of the IEEE.

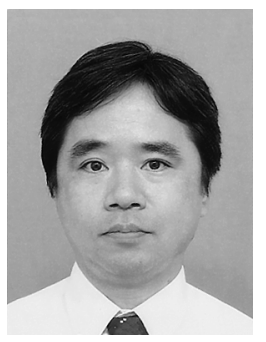

Hidekazu Murata received the B.E., M.E., and Ph.D. degrees in electronic engineering from Kyoto University, Kyoto, Japan, in 1991, 1993, and 2000, respectively. In 1993, he joined the Faculty of Engineering, Kyoto University. From 2002 to 2006, he was an Associate Professor of Tokyo Institute of Technology. He has been at Kyoto University since October 2006 and is currently an Associate Professor of Department of Communications and Computer Engineering, Graduate School of Informatics. His major research interests include signal processing and its hardware implementation, with particular application to cooperative wireless networks with cognitive radio capabilities. He received the Young Researcher's Award from the IEICE of Japan in 1997, the Ericsson Young Scientist Award in 2000, and the Young Scientists' Prize of the Commendation for Science and Technology by the Minister of Education, Culture, Sports, Science and Technology in 2006. He is a member of the IEEE and SITA.

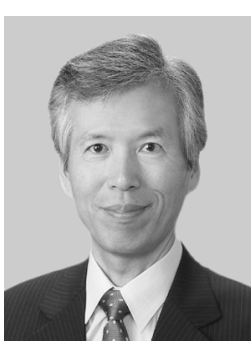

Susumu Yoshida received the B.E., M.E. and Ph.D. degrees all in electrical engineering from Kyoto University, Kyoto, Japan in 1971, 1973 and 1978, respectively. Since 1973, he has been with the Faculty of Engineering, Kyoto University and currently he is a full professor of the Graduate School of Informatics, Kyoto University. During the last 30 years, he has been mainly engaged in the research of wireless personal communications. His current research interest includes highly spectrally efficient wireless transmission techniques and distributed controlled wireless networks. During 1990-1991, he was a visiting scholar at WINLAB, Rutgers University, U.S.A. and Carleton University in Ottawa, Canada. He served as a TPC Chair of IEEE VTC 2000-Spring, Tokyo. He was a guest editor of IEEE J-SAC on Wireless Local Communications published in April and May 1996. He received the IEICE Achievement Award and Ericsson Telecommunication Award in 1993 and 2007, respectively. 